\title{
Precipitating Factors and Treatment Outcomes of Hepatic Encephalopathy in Liver Cirrhosis
}

Nandu S. Poudyal ${ }^{1}$, Sitaram Chaudhary ${ }^{1}$, Sudhamshu KC ${ }^{2}$, Bidhan N. Paudel ${ }^{1}$, Bhupendra K. Basnet $^{3}$, Amrendra Mandal ${ }^{4}$, Paritosh Kafle ${ }^{4}$, Baikuntha Chaulagai ${ }^{4}$, Azad Mojahedi ${ }^{5}$, Mukesh S. Paudel ${ }^{6}$, Barun Shrestha ${ }^{7}$, Vijay Gayam ${ }^{4}$

1. Gastroenterology, National Academy of Medical Sciences, Kathmandu, NPL 2. Hepatology, National Academy of Medical Sciences, New Delhi, IND 3. Gastroenterology, National Academy of Medical Sciences, Kathamandu, NPL 4. Internal Medicine, Interfaith Medical Center, Brooklyn, USA 5. Internal Medicine, Interfaith Medical Centre, Brooklyn, USA 6. Gastroenterology, Lumbini City Hospital, Patan, NPL 7. Gastroenterology, Chitwan Medical College, Bharatpur, NPL

$\square$ Corresponding author: Nandu S. Poudyal, nandupoudyal@gmail.com

Disclosures can be found in Additional Information at the end of the article

\section{Abstract}

\section{Background}

Hepatic encephalopathy (HE) is a common cause of hospital admission in patients with liver cirrhosis (LC). The aims of this study were to evaluate the precipitant factors and analyze the treatment outcomes of HE in LC.

\section{Methods}

All the LC patients admitted between February 2017 and January 2018 for overt HE were analyzed for precipitating factors and treatment outcomes. Treatments were compared among three treatment groups: receiving lactulose, lactulose plus L-ornithine L-aspartate (LOLA), and lactulose plus rifaximin. The primary endpoints were mortality and hospital stay. The chisquare test was used to compare the different treatment outcomes with hospital stay and mortality with significance at $\mathrm{p}<0.05$.

\section{Results}

A total of 132 patients (mean age $49.2 \pm 10.2$ years; male/female ratio of 103:29) were studied. The most common precipitating factor of HE was infection 65 (49.2\%), followed by electrolyte imbalance 54 (41\%), constipation 44 (33.33\%), and gastrointestinal bleeding 21 (16\%) patients. At the time of admission, 29 (22\%), 76 (57.5\%), 21 (16\%), and six (4.5\%) patients had grade I, II, III, and IV HE, respectively. The difference in mortality was not statistically significant $(p=0.269)$ in three groups but the hospital stay was shorter among patients in groups B and C than in group A alone $(7.36 \pm 4.58$ and $7 \pm 3.69,9.64 \pm 5.28$ days, respectively, $\mathrm{p}=0.015)$.

\section{Conclusions}

Infection, especially spontaneous bacterial peritonitis, was the commonest precipitating factor of HE. The combination of lactulose either with LOLA or rifaximin is equally effective in improving HE and reducing the duration of hospital stay than lactulose alone. 
Categories: Internal Medicine, Neurology, Gastroenterology

Keywords: hepatic encephalopathy, liver cirrhosis, precipitating factors, treatment outcome

\section{Introduction}

Liver cirrhosis (LC), the final pathway for a wide variety of chronic liver diseases, is a pathologic entity defined as diffuse hepatic fibrosis with the replacement of the normal liver architecture by nodules [1]. Hepatic encephalopathy (HE) is a well-recognized clinical complication of LC and the presence and prompt identification of well-defined precipitating factors are extremely important in the diagnosis and treatment of this fatal condition.

HE develops in $50 \%$ to $70 \%$ of patients with cirrhosis, and its occurrence is a poor prognostic indicator, with projected one- and three-year survival rates of $42 \%$ and $23 \%$, respectively, without liver transplantation [2].

Lactulose is commonly used in the treatment of HE, however, there is increasing evidence of add-on therapy with rifaximin. Additionally, there is no study conducted using L-ornithine Laspartate (LOLA) plus lactulose with lactulose alone in our knowledge. We aimed to study the different combinations of these drugs in our population with lactulose in all the three groups as a standard of care.

\section{Materials And Methods}

A descriptive, cross-sectional, single-center study was carried out on 132 consecutive patients of HE admitted to the department of gastroenterology and liver unit at National Academy of Medical Sciences, Bir Hospital, Nepal, between February 2017 and January 2018. All patients ages ranging from 18 to 75 years with LC with HE type C, irrespective of the etiology of LC, were enrolled while LC patients with intractable HE or other identifiable brain lesions, LC with other co-morbid conditions, and death within 24 hours were excluded from the study.

LC was diagnosed on the basis of the clinical examination and biochemical and imaging diagnosis. The diagnosis of acute HE was made on the basis of a detailed history and physical examination and West Haven Criteria [3]. The clinical findings on examination, including the presence of jaundice, pallor, fever, asterixis, and ascites, were recorded. Improvement in HE was defined as the complete reversal of clinical symptoms on the basis of the West Haven Criteria.

Blood samples were sent for complete blood count, random blood sugar, liver function tests, renal function tests, serum albumin levels, hepatitis B virus (HBV), anti-hepatitis C virus (antiHCV), human immunodeficiency virus (HIV), coagulation profile, urine analysis, and blood cultures. Findings of abdominal ultrasound, including liver and spleen size, parenchymal echogenicity, portal vein diameter, and ascites were noted. An ascitic tap was done in all patients with ascites and sent for a detailed report and culture in order to diagnose infection on ascites. Urine routine and culture were also sent in all patients, along with blood culture, in order to diagnose any infection. A chest X-ray was done in all patients on arrival at the hospital. Non-contrast computed tomography (CT) head was also done to exclude intracranial lesions, especially bleed. All other supportive investigations were done as and when needed.

After meeting the inclusion criteria, patients were divided into three groups. Group A $(n=44)$ patients were treated with lactulose alone as a syrup formulation, containing $40 \mathrm{~g}$ lactulose/60 $\mathrm{mL}, 30-60 \mathrm{ml}$, three times a day, to ensure the patient passes two to three semi-soft stools in a day. Group B $(n=44)$ patients were treated with lactulose and LOLA (intravenous 20 grams per day) until the patients reverted back to normal. They were then given a $5 \mathrm{~g}$ sachet formulation containing granules with a dose of $20 \mathrm{~g} /$ day until hospital stay. Group C ( $\mathrm{n}=44)$ was treated with 
lactulose and rifaximin, $550 \mathrm{mg}$ capsule two times a day. If the patients did not improve with the given treatment or if another drug had to be added to improve their condition, those patients were included as treatment failures. Patients were followed until they were discharged from the hospital or died during hospital stay. So the primary endpoints were mortality and hospital stay.

Patients also received other standard treatments according to their need, which included antibiotics according to the sensitivity of the culture report, electrolyte correction, and control of gastrointestinal bleed (GIB) by vasopressin analogs (octreotide or terlipressin) as initial medical management before definitive therapy with endoscopy was performed.

\section{Data analysis and statistical methods}

The data obtained from the study were analyzed using Statistical Package for Social Studies (SPSS) version 17 (IBM Corp., Armonk, NY, US). For a comparison of categorical variables, the chi-square test, independent t-test, and bivariate and multivariate analyses were used to compare the results of various parameters among the studied patients. Values were expressed as mean $\pm \mathrm{SD}$, a $95 \%$ confidence interval was taken, and $p$-values of $<0.05$ were considered statistically significant.

\section{Results}

A total of 140 consecutive patients with LC and HE were enrolled. Of these, eight patients were excluded because of significant systemic illness (acute respiratory distress syndrome (ARDS), septic shock) and two patients did not give consent. Finally, $132(n=132)$ cases were included in the study, as shown in the flow chart in Figure 1.

140 patients Enrolled

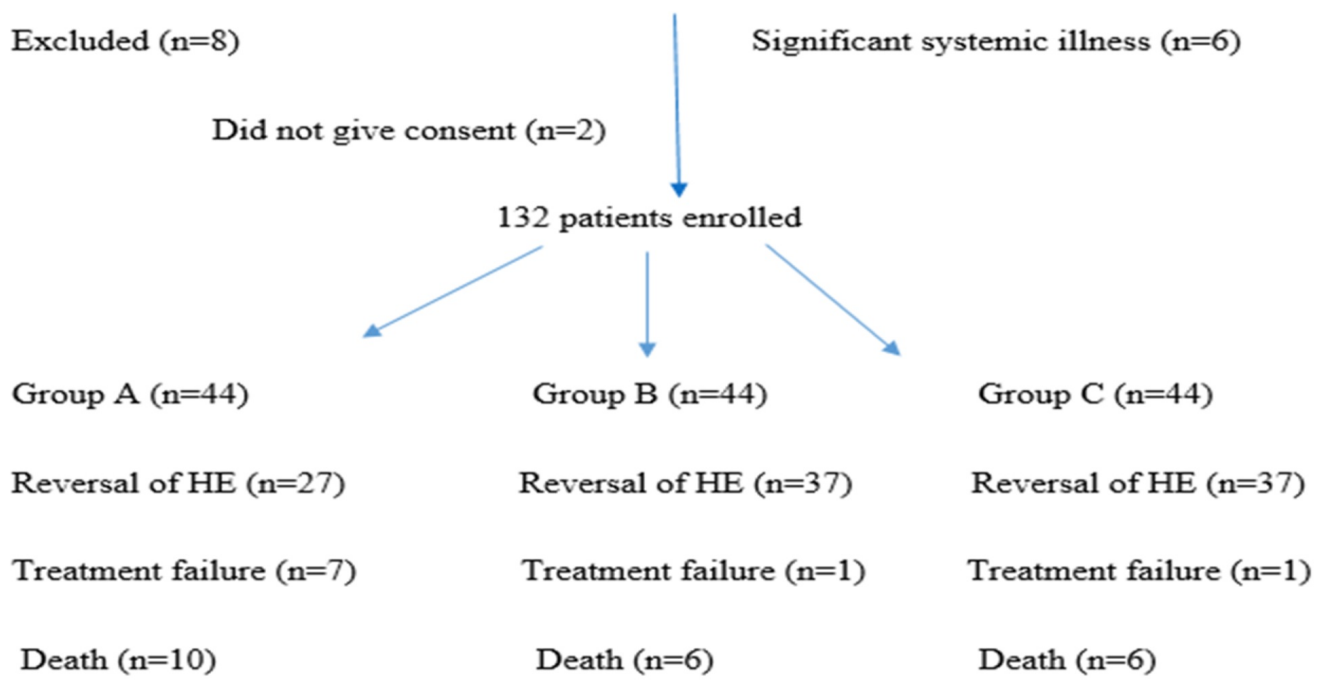

FIGURE 1: Flowchart on types of cases included in the study

The most common cause of chronic liver disease was found to be alcohol - 114 (86\%), followed by hepatitis B virus - nine (7\%), hepatitis C virus - six (5\%), and cryptogenic - three (2\%) in our study. To assess the severity of liver disease in our patients, Child-Pugh (CTP), model endstage liver disease (MELD), MELD-Na scoring, and HE grading were applied. In this study, 38 (28.8\%) patients were in CTP class B and 94 (71.2 \%) were in CTP class C. The mean CTP score was $10.24 \pm 1.85$ and the mean MELD score was $24.5 \pm 4.2$. Twenty-nine (22\%) patients had 


\section{Cureus}

grade I, 76 (57.5\%) had grade II, 21 (16\%) had grade III, and six (4.5\%) had grade IV HE at the time of admission (Table 1 ).

\begin{tabular}{|l|llll|}
\hline Parameters & Lactulose & Lactulose + Rifaximin & Lactulose + LOLA & p-value \\
\hline Age (Years) & $48.68 \pm 9.00$ & $48.20 \pm 10.69$ & $50.73 \pm 10.86$ & 0.471 \\
Male/Female & $29: 15$ & $37: 7$ & $37: 7$ & \\
Etiology & & & $38(86.3 \%)$ & \\
Alcohol & $39(88.6 \%)$ & $36(81.8 \%)$ & $3(6.8 \%)$ & \\
HBV & $3(6.8 \%)$ & $3(6.8 \%)$ & $2(4.6 \%)$ & \\
HCV & $1(2.3 \%)$ & $3(6.8 \%)$ & $1(2.3 \%)$ & 0.084 \\
Others & $1(2.3 \%)$ & $2(4.6 \%)$ & $11(25 \%)$ & 0.024 \\
CTP B & $9(20.5 \%)$ & $18(40.9 \%)$ & $33(75 \%)$ & 0.112 \\
CTP C & $35(79.5 \%)$ & $26(59.1 \%)$ & $21.98 \pm 7.78$ & 0.02 \\
MELD score & $24.50 \pm 8.02$ & $20.16 \pm 6.20$ & $24.02 \pm 8.37$ & $6 / 27 / 6 / 5$ \\
\hline MELD Na & $26.61 \pm 7.79$ & $23.27 \pm 7.16$ & & \\
HE grade 1/2/3/4 & $16 / 21 / 7 / 0$ & $7 / 28 / 8 / 1$ & & \\
\hline
\end{tabular}

\section{TABLE 1: Baseline characteristics of study patients}

LOLA: L-ornithine L-aspartate; HBV: hepatitis B virus; HCV: hepatitis C virus; CTP: Child-Pugh; MELD: model for end-stage liver disease; HE: hepatic encephalopathy

Baseline total count, hemoglobin, platelets, blood sugar random, prothrombin time, international normalized ratio (INR), total protein, albumin, liver function test, renal function test, and serum electrolyte were comparable in the three groups (Table 2). 


\section{Cureus}

\begin{tabular}{|c|c|c|c|c|}
\hline Parameters & Lactulose & $\begin{array}{l}\text { Lactulose and } \\
\text { LOLA }\end{array}$ & $\begin{array}{l}\text { Lactulose and } \\
\text { Rifaximin }\end{array}$ & p-value \\
\hline Total Count (/cumm), mean \pm SD & $12693.00 \pm 7903.59$ & $11348.00 \pm 10933.47$ & $10639.00 \pm 8206.84$ & 0.563 \\
\hline Hemoglobin (gm/dL), mean \pm SD & $9.16 \pm 1.85$ & $9.48 \pm 2.46$ & $9.67 \pm 2.21$ & 0.549 \\
\hline Platelets (/cumm), mean \pm SD & $118890 \pm 133268.00$ & $101970 \pm 55181.56$ & $116670.00 \pm 53739.14$ & 0.625 \\
\hline Blood sugar (mg/dl), mean \pm SD & $99.02 \pm 30.60$ & $116.39 \pm 47.45$ & $117.02 \pm 105.85$ & 0.387 \\
\hline Prothombin_time (secs), mean \pm SD & $23.23 \pm 8.93$ & $22.87 \pm 10.51$ & $23.65 \pm 9.81$ & 0.932 \\
\hline INR, mean \pm SD, & $1.91 \pm 0.72$ & $1.85 \pm 0.67$ & $1.92 \pm 0.70$ & 0.885 \\
\hline Total protein (gm/dL), mean $\pm S D$ & $6.23 \pm 1.03$ & $6.25 \pm 0.87$ & $6.46 \pm 1.04$ & 0.480 \\
\hline Albumin (gm/dL), mean $\pm S D$ & $2.45 \pm 0.52$ & $2.53 \pm 0.50$ & $2.44 \pm 0.54$ & 0.670 \\
\hline Bilirubin total $(\mathrm{gm} / \mathrm{dL})$, mean $\pm \mathrm{SD}$ & $11.30 \pm 10.43$ & $9.13 \pm 8.86$ & $6.51 \pm 7.26$ & 0.046 \\
\hline Bilirubin direct (gm/dL), mean $\pm \mathrm{SD}$ & $6.71 \pm 6.48$ & $4.72 \pm 5.35$ & $3.37 \pm 4.39$ & 0.018 \\
\hline AST (IU/L), mean $\pm S D$ & $55.68 \pm 34.5$ & $50.84 \pm 31.77$ & $65.02 \pm 70.21$ & 0.385 \\
\hline AST (IU/L), mean $\pm S D$ & $122.11 \pm 90.96$ & $102.66 \pm 56.84$ & $126.36 \pm 129.89$ & 0.478 \\
\hline $\begin{array}{l}\text { Alkaline phosphatase (IU/L), } \\
\text { mean } \pm S D\end{array}$ & $114.57 \pm 64.94$ & $125.20 \pm 68.27$ & $134.68 \pm 67.70$ & 0.373 \\
\hline Sodium (Meq/l) & $132.07 \pm .12$ & $129.89 \pm 9.94$ & $132.34 \pm 10.35$ & 0.397 \\
\hline Potassium (M eq/I), mean $\pm S D$ & $4.16 \pm 1.07$ & $4.05 \pm 0.94$ & $4.42 \pm 0.84$ & 0.182 \\
\hline Urea (gm/dl) & $67.48 \pm 50.33$ & $47.86 \pm 27.58$ & $69.68 \pm 41.23$ & 0.025 \\
\hline Creatinine (gm/dl), mean $\pm S D$ & $1.63 \pm 1.25$ & $0.97 \pm 0.34$ & $1.62 \pm 1.10$ & 0.002 \\
\hline
\end{tabular}

\section{TABLE 2: Baseline laboratory parameters of the study patients}

INR: international normalized ratio; AST: aspartate aminotransferase

\section{Precipitating factors for hepatic encephalopathy}

Out of 132 patients, infection was the most common factor seen in 65 (49.2\%) patients in this study. Infection in the form of spontaneous bacterial peritonitis (SBP) (18.2\%) was the most common precipitant factor followed by $14.4 \%$ respiratory tract infections, $13.7 \%$ urinary tract infections, and 3\% with fever of undetermined cause. Dyselectrolytemia was present in 54 (41\%) patients while 44 (33.33\%) patients were having constipation and 11 (16\%) patients had GIB (Table 3). 


\section{Cureus}

\begin{tabular}{lllll}
\hline Precipitating factors & Group A $(\mathbf{n = 4 4 )}$ & Group B (n=44) & Group C (n=44) \\
\hline Spontaneous bacterial peritonitis, $n(\%)$ & $7(15.9)$ & $7(15.9)$ & $10(22.7)$ \\
\hline Respiratory tract infection, $\mathrm{n}(\%)$ & $6(13.6)$ & $8(18.2)$ & $5(11.4)$ \\
\hline Urinary tract infection, $\mathrm{n}(\%)$ & $8(18.2)$ & $7(15.9)$ & $3(6.8)$ \\
\hline Fever of unknown origin, $\mathrm{n}(\%)$ & $1(2.3)$ & $1(2.3)$ & $2(4.5)$ \\
Dyselectrolytemia, $\mathrm{n}(\%)$ & $20(45.5)$ & $18(40.9)$ & $16(36.3)$ \\
Constipation, $\mathrm{n}(\%)$ & $12(27.3)$ & $14(31.8)$ & $18(40.9)$ \\
Gastrointestinal bleeding, $\mathrm{n}(\%)$ & $9(20.5)$ & $6(13.6)$ & $6(13.6)$
\end{tabular}

TABLE 3: Precipitating factors of study patients with hepatic encephalopathy

\section{Evaluation of outcome}

The mean hospital stay in the study population was $9.64 \pm 5.28,7.36 \pm 4.58$, and $7 \pm 3.69$ in group $A$, group $B$, and group $C$, respectively $(\mathrm{p}=0.015)$. So patients in the lactulose plus LOLA and lactulose plus rifaximin groups had a shorter hospital stay as compared with the lactulosealone group. Kaplan-Meier survival (Figure 2) for the three treatment groups showed that survival during the hospital stay was highest for the group, which received lactulose and LOLA, followed by the group with lactulose plus rifaximin. Survival was lowest for the group that received lactulose only.

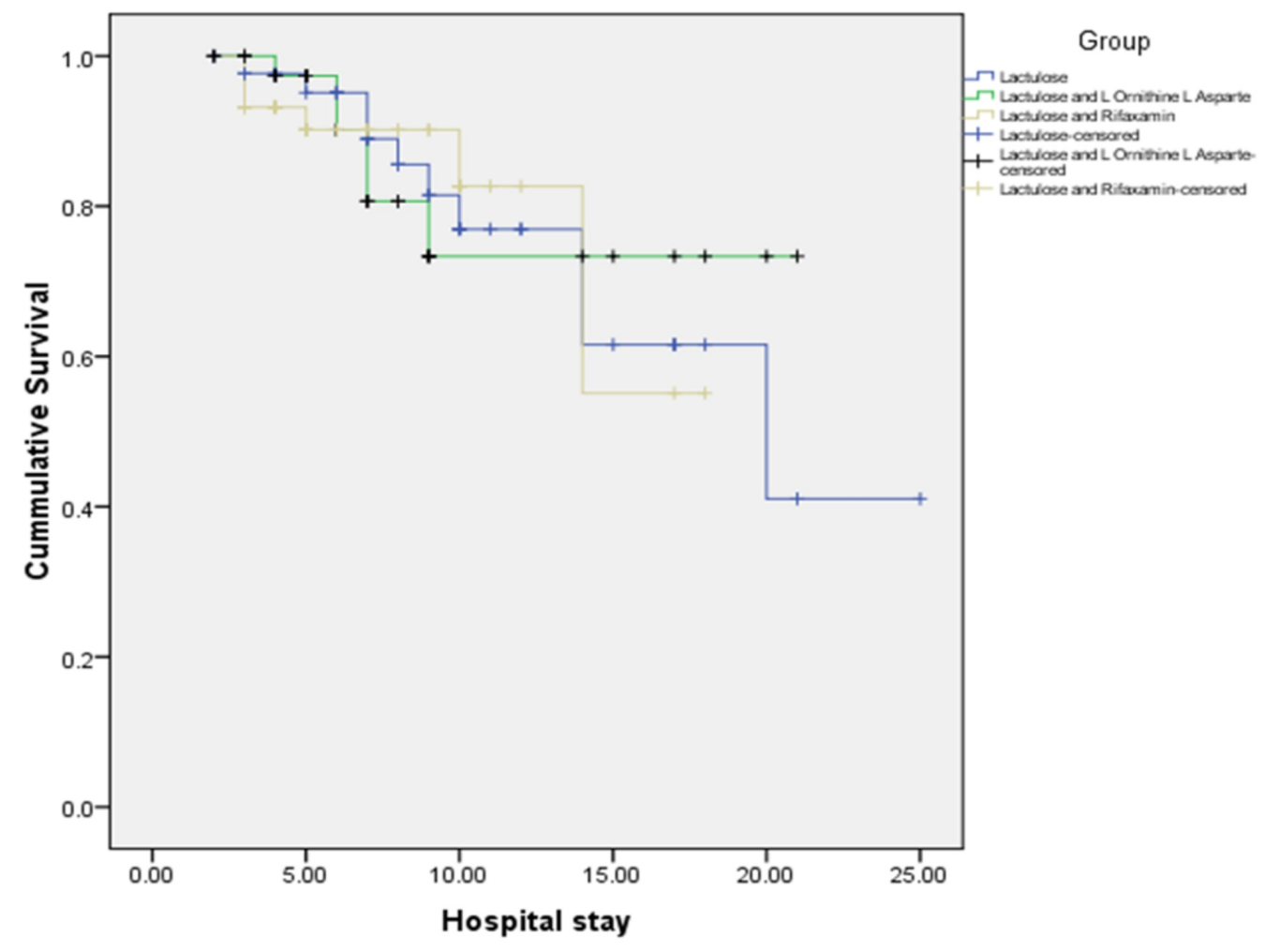

FIGURE 2: Kaplan-Meier survival curve for the three treatment 


\section{Treatment response}

In group A, 27 (61.4\%) patients fully recovered from HE with lactulose only, in group B, 37 (84.1\%) patients fully recovered from HE with lactulose and LOLA, and in group C too, 37 (84.1\%) patients had a complete reversal of HE. This difference was statistically significant $(p=0.017)$. There was a decrease in mortality in the lactulose plus rifaximin group and lactulose plus LOLA group with six (13.6 \%) each in groups A and B vs. lactulose alone 10 (22.7\%) with pvalue $<0.269$. Out of 132 patients enrolled in our study, mortality was seen in $22(16.7 \%)$ patients, and it was found that the mortality rate was significantly higher in child class $\mathrm{C}$ patients.

Predictors of non-response to therapy in all three groups had a higher MELD score (30.59 \pm 7.25 vs. $20.54 \pm 6.41)$, higher Child score $(12.32 \pm 1.29$ vs. $9.83 \pm 1.74)$, and baseline higher total leukocyte count $(17482 \pm 9668.6$ vs $10375 \pm 8529.14)$ as compared to the patients who responded $(\mathrm{p}<0.001)$.

\section{Discussion}

The identification and treatment of the precipitating factor is the most important aspect of the management of acute HE since symptoms of overt HE are debilitating and lead to nonadherence to a therapeutic regimen, which, in turn, leads to severe symptoms, frequent hospitalizations, and poor quality of life [4].

There had been some differences in the common precipitation factors across the studies. SBP was the most common precipitating factor reported in our study. This was consistent with a study done in Pakistan by Mumtaz et al. and Abid et al. who reported SBP as the most common precipitant in HE [5-6]. Our finding is, however, contradicting studies done in the USA by Souheil et al., who observed that infections were responsible in only $3 \%$ of cases. This could be related to adherence to therapy and regular monitoring of patients with LC in the USA, which resulted in early detection and treatment of infections [7].

In our study, we observed electrolyte imbalance to be present in $41 \%$ patients, and among electrolyte disorders, hyponatremia was much more common than hypokalemia in our patients, which was in agreement with other studies, such as Abid et al. and Alam et al. [6-8].

Constipation was seen in $33.7 \%$ in our study, which was consistent with the study by Abid et al. who reported that $21.7 \%$ of patients had constipation. However, our finding contradicts the study done by Zakaria et al., who reported constipation as a precipitating factor in only $7 \%$ of patients. Gastrointestinal bleeding (GIB) was identified only in 16\% in our study, which is similar to the study done by Souheil et al., who also reported GIB in $18 \%$ of cases [7,9]. This was contrary to the findings of Bustamante et al. and Mehboob et al., who reported that GIB was the second and third commonest precipitating factor, respectively [10-11]. This can be attributed to a larger number of patients with large varices, which carry the risk of variceal bleed. The intake of a large amount of protein diet, especially meat and meat products, was also found in $16 \%$ patients as a precipitant factor in our study, which is similar to the study done by Devrajani et al. Similar to Mumtaz et al.'s study, the majority of our patients were grade II HE [5,12].

Lactulose, a non-absorbable disaccharide inhibiting intestinal ammonia production by lowering the colonic $\mathrm{pH}$, is currently recommended as the first-line pharmacological treatment for HE [13]. In our study, only $61.4 \%$ of patients fully recovered from HE with lactulose alone and 
22.7\% had mortality. LOLA, a stable salt of the natural LOLA and aspartic acid, has been shown to improve HE [14]. Abdo-Francis et al. observed that treatment with LOLA was more effective than lactulose in improving HE, which is similar to our study [15]. However, in our study, we used LOLA plus lactulose in group B in which $84.1 \%$ of patients reverted back to normal. Only $2.3 \%$ of patients had treatment failure and $13.6 \%$ of patients died in the lactulose plus LOLA group. To our knowledge, there are no other studies comparing the combination of LOLA and lactulose with lactulose alone. Therefore, more studies are required to derive optimal results. However, LOLA infusion appears to be a safe and effective treatment of HE in our study.

Our study showed the superiority of rifaximin plus lactulose therapy over treatment with lactulose alone. We found that recovery from HE was $84 \%$ in group $\mathrm{C}$, which is similar to the study done by Sharma et al., which showed that the combination of lactulose plus rifaximin is more effective than lactulose alone in the treatment of overt HE [16]. The risk of bacterial resistance appears to be lower with rifaximin than with systemic antibiotics. The plasma levels of rifaximin are negligible; therefore, bacteria outside the gastrointestinal tract are not exposed to appreciable selective pressure [17]. Thus, Rifaximin may be used in the long term or can be used several times during overt HE.

Our study is also supported by Bass et al., who also showed the superiority of rifaximin therapy over treatment with lactulose alone. More than $90 \%$ of patients received concomitant lactulose during the study period, and a significant treatment effect was noted [18]. Regardless of the decrease in mortality in the lactulose plus rifaximin group and the lactulose plus LOLA group in our study, it was not significant. However, Sharma et al. observed a significant decrease in mortality after treatment with lactulose plus rifaximin vs. lactulose and placebo [16].

The limitations of our study include it involving a single center and the small sample size. Further, large-scale, multicenter trials should be evaluated using robust clinical outcomes.

\section{Conclusions}

Infection, especially SBP, is the commonest precipitating factors of HE followed by electrolyte imbalance, constipation, and GIB. The combination of lactulose either with LOLA or rifaximin is equally more effective in improving $\mathrm{HE}$ and reducing the duration of hospital stay than lactulose alone.

\section{Additional Information \\ Disclosures}

Human subjects: Consent was obtained by all participants in this study. National Academy of Medical Sciences issued approval 733/2073/74. Animal subjects: All authors have confirmed that this study did not involve animal subjects or tissue. Conflicts of interest: In compliance with the ICMJE uniform disclosure form, all authors declare the following: Payment/services info: All authors have declared that no financial support was received from any organization for the submitted work. Financial relationships: All authors have declared that they have no financial relationships at present or within the previous three years with any organizations that might have an interest in the submitted work. Other relationships: All authors have declared that there are no other relationships or activities that could appear to have influenced the submitted work.

\section{References}

1. Fitz JG: Hepatic encephalopathy, hepatorenal syndrome, hepatopulmonary syndrome and other systemic complications of liver. Gastrointestinal and Liver Disease. Feldman M, Friedman LS, Sleisenger MH (ed): Elsevier, Philadelphia; 2016. 1577-1590. 
2. Bustamante J, Rimola A, Ventura PJ, Navasa M, Cirera I, Reggiardo V: Prognostic significance of hepatic encephalopathy in patients with cirrhosis. J Hepatol. 1999, 30:890-895.

10.1016/S0168-8278(99)80144-5

3. Ferenci P, Lockwood A, Mullen K, Tarter R, Weissenborn K, Blei AT: Hepatic encephalopathy -definition nomenclature, diagnosis, and quantification: final report of the working party at the 11th World Congresses of Gastroenterology, Vienna, 1998. Hepatology. 2002, 35:716-721. 10.1053/jhep.2002.31250

4. Blei AT, Bircher J, Benhamou JP, McIntyre N, Rizzetto M, Rodés J: Hepatic encephalopathy. Oxford Textbook of Clinical Hepatology. Neil McIntyre, Jean-Pierre Benhamou, Johannes Bircher, Mario Rizzetto, Juan Rodes (ed): Oxford Medical, Oxford; 1998. 765-783.

5. Mumtaz K, Ahmed US, Abid S, Baig N, Hamid S, Jafri W: Precipitating factors and the outcome of hepatic encephalopathy in liver cirrhosis. J Coll Physicians Surg Pak. 2010, 20:514-518.

6. Abid S, Jafri W, Mumtaz K, Islam M, Abbas Z, Shah HA: Efficacy of L-ornithine-L-aspartate as an adjuvant therapy in cirrhotic patients with hepatic encephalopathy. J Coll Physicians Surg. 2011, 21:666-671.

7. Abou-Assi S, Vlahcevic ZR: Hepatic encephalopathy. Metabolic consequence of cirrhosis often is reversible. Postgrad Med. 2001, 109:52-70. 10.3810/pgm.2001.02.850

8. Alam I, Razaullah HI, Humayun M, Taqweem MA, Nisar M: Spectrum of precipitating factors of hepatic encephalopathy in liver cirrhosis. Pak J Med Res. 2005, 44:96-100.

9. Zakaria M, UR-Rehman M, But A: Precipitating factors of hepatic encephalopathy among Sudanese patients with liver cirrhosis. Professional Med J. 2008, 15:375-379.

10. Bustamante J, Rimola A, Ventura PJ, Navasa M, Cirera I, Reggiardo V, Rodés J: Prognostic significance of hepatic encephalopathy in patients with cirrhosis. J Hepatol. 1999, 30:890-895. 10.1016/S0168-8278(99)80144-5

11. Mahboob F: Frequency of risk factors for hepatic encephalopathy in patients of chronic liver disease. Ann King Edw Med Univ. 2003, 9:29-30. 10.21649/akemu.v9i1.1313

12. Devrajani BR, Shah SZA, Devrajani T, Kumar D: Precipitating factors of hepatic encephalopathy at a tertiary care hospital. J Pak Med Assoc. 2009, 59:683.

13. Vilstrup H, Amodio P, Bajaj J, et al.: Hepatic encephalopathy in chronic liver disease: 2014 practice guideline by the American Association for the Study of Liver Diseases and the European Association for the Study of the Liver. Hepatology. 2014, 60:715-735. 10.1002/hep.27210

14. Gebhardt R, Beckers G, Gaunitz F, Haupt W, Jonitza D, Klein S: Treatment of cirrhotic rats with 1-ornithine-l-aspartate enhances urea synthesis and lowers serum ammonia levels. J Pharmacol Exp Ther. 1997, 283:1-6.

15. Abdo-Francis J, Perez-Hernandez J, Hinojosa-Ruiz A, Hernandez-Vasquez J: Use of L-ornitin L-aspartate (LOLA) reduces time of hospital stay in patients with hepatic encephalopathy [Article in Spanish]. Rev Gastroenterol Mex. 2010, 75:135-141.

16. Sharma BC, Sharma P, Lunia MK, Srivastava S, Goyal R, Sarin SK: A randomized double-blind, controlled trial comparing rifaximin plus lactulose with lactulose alone in treatment of overt hepatic encephalopathy. Am J Gastroenterol. 2013, 108:1458-1463.

17. Debbia E, Maioli E, Roveta S, Marchese A: Effects of rifaximin on bacterial virulence mechanisms at supra-and sub-inhibitory concentrations. J Chemother. 2008, 20:186-194. 10.1179/joc.2008.20.2.186

18. Bass NM, Mullen KD, Sanyal A, et al.: Rifaximin treatment in hepatic encephalopathy. N Engl J Med. 2010, 362:1071-1081. 10.1056/NEJMoa0907893 